\title{
FINITE ELEMENT MODEL DEVELOPED AND MODAL ANALYSIS OF LARGE SCALE STEAM TURBINE ROTOR: QUANTIFICATION OF UNCERTAINTIES AND MODEL UPDATING
}

\author{
Dimitrios Giagopoulos $^{1}$, Alexandros Arailopoulos ${ }^{1}$, Ilias Zacharakis ${ }^{1}$, Eleni Pipili ${ }^{1}$ \\ ${ }^{1}$ Department of Mechanical Engineering, University of Western Macedonia \\ Kozani 50100, Greece \\ e-mail: dgiagopoulos@uowm.gr, aarailopoulos@uowm.gr,liakos_z@hotmail.com, \\ elenipipili92@gmail.com
}

Keywords: Structural Dynamics, Large scale structures, Modal identification, Model updating, Substructuring, Rotordynamics.

\begin{abstract}
.
In this work, the effectiveness of a computational framework to handle large scale linear and nonlinear models is presented by calibrating a high-fidelity FE model of a steam turbine rotor with several millions of degrees of freedom, using experimentally identified modal parameters. An extensible framework for Bayesian Uncertainty Quantification and Propagation of complex and computationally demanding physical models, was connected in an efficient way with a numerical code leading to an automated determination of dynamic response of linear and nonlinear mechanical systems. The effect of correlation in the prediction error models postulated in the Bayesian model selection and parameter estimation technique is investigated. First, using an integrated reverse engineering strategy, the digital shape of the three sections of a steam turbine rotor was developed and the final parametric CAD model was created. The finite element model of the turbine were created using tetrahedral solid elements. Due to complex geometry of the structure, the developed model consists of about fifty-five million DOFs. The identification of modal characteristics of the frame is based on acceleration time histories, which are obtained through an experimental investigation of its dynamic response in a support-free state by imposing impulsive loading. The developed computational framework with appropriate substructuring methods, are used for estimating the parameters (material properties) of the finite element model, based on minimizing the deviations between the experimental and analytical modal characteristics (modal frequencies and mode shapes). Direct comparison of the numerical and experimental data verified the reliability and accuracy of the methodology applied. The identified finite element model is representative of the initial structural condition of the turbine and is used to develop a simplified finite element model, which then used for the turbine rotordynamic analysis. Also, this model can be further used for structural health monitoring purposes of the rotor.
\end{abstract}




\section{INTRODUCTION}

Reverse engineering is a modern field of engineering, which finds application in many areas of industry. Current industrial design requirements, tend to have the need of improving, modifying and developing new and optimized versions of various mechanical parts or even entire structures. For many of these structures, there is no available information not only about their geometric and designing details, but also about their material properties and mechanical treatment and procedures carried out during the construction process. To address this issue, an integrated reverse engineering strategy is necessary to be applied [1-5]. In this process, many issues are taken into account, related to the development of the geometry and finite element model, with the experimental modal analysis procedures and the application of robust and effective computational model updating techniques. The main objective of the present work is to demonstrate the advantages of a developed computational framework to handle large scale linear and nonlinear models, by calibrating a high-fidelity FE model of a steam turbine rotor with several millions of degrees of freedom, using experimentally identified modal parameters.

Applying classical finite element techniques, the equations of motion of mechanical systems with complex geometry are first set up. As the order of these models increases, the existing numerical and experimental methodologies for a systematic determination of their dynamic response become inefficient to apply. Therefore, there is a need for the development, improvement and application of new suitable methodologies for investigating dynamics of large scale mechanical models in a systematic and efficient way. Traditionally, in the area of structural dynamics this is done by first employing methodologies that reduce the dimensions of the original system. In this paper examined a time domain reduction method [1-5]. In order to improve the FE model of the structure, structural model updating techniques [16], have been proposed in order to reconcile the numerical (FE) model, with experimental data. Structural model parameter estimation based on measured modal data (e.g. [6-12]) are often formulated as weighted least-squares estimation problems in which metrics, measuring the residuals between measured and model predicted modal characteristics, are build up into a single weighted residuals metric formed as a weighted average of the multiple individual metrics using weighting factors. Standard gradient-based optimization techniques are then used to find the optimal values of the structural parameters that minimize the single weighted residuals metric representing an overall measure of fit between measured and model predicted modal characteristics.

The accuracy of the predictions will be uncertain, on the one hand, because of the uncertainty of all future structural excitations and on the other hand, because the structural model will always involve approximations of the real dynamic behavior that affects in an uncertain manner the predicted responses. Uncertainties related to model-structure errors, arise from the assumptions made to parameterize and describe the behaviour of the physical structure, because of simplifications and erroneous assumptions, inexact modeling of the material constitutive behaviour, inexact modeling of boundary conditions, errors because of the spatial discretization of the distributed structural system, unmodeled features such as neglected nonstructural components, as well as errors introduced by numerical methods. Such uncertainties, in this work, are treated meticulously and are minimized to the most plausible extent according to each problem, as cannot be regarded and tuned by model updating methodologies. Model updating basically regards, erroneous assumptions of model parameters such as material parameters (Young's modulus and mass density), cross section properties (moments of inertia), shell or plate thickness, spring stiffnesses and non-structural mass.

The organization of this paper is as follows. First, in the following section, is presented an overview of the formulation for finite element model updating based on modal data. In the third 
section, the structure examined (steam turbine) is introduced. More specifically, first presented the procedure followed in order to develop the digital shape of the steam turbine (rotor and blades), using a 3D Laser Scanner. The development of the detailed FE model of the structure analyzed next and finally is given a brief review of the experimental modal analysis results. Finally, the parametric studies on updating finite element model of the system are presented in the fourth section. Conclusions are summarized in the fifth section.

\section{FINITE ELEMENT MODEL UPDATING METHOD}

Let $D=\left\{\hat{\omega}_{r}, \underline{\hat{\phi}_{r}} \in R^{N_{o}}, r=1, \ldots, m\right\}$ be the measured modal data from a structure, consisting of modal frequencies $\hat{\omega}_{r}$ and mode shape components $\underline{\hat{\phi}}_{r}$ at $N_{o}$ measured DOFs, where $m$ is the number of observed modes. Consider a parameterized class of linear structural models used to model the dynamic behavior of the structure and let $\underline{\theta} \in R^{N_{\theta}}$ be the set of free structural model parameters to be identified using the measured modal data. The objective in a modal-based structural identification methodology is to estimate the values of the parameter set $\underline{\theta}$ so that the modal data $\left\{\omega_{r}(\underline{\theta}), \underline{\phi_{r}}(\underline{\theta}) \in R^{N_{0}}, r=1, \ldots, m\right\}$ predicted by the linear class of models at the corresponding $N_{0}$ measured DOFs best matches the experimentally obtained modal data in $D$. For this, let

$$
\varepsilon_{\omega_{r}}(\underline{\theta})=\frac{\omega_{r}^{2}(\underline{\theta})-\hat{\omega}_{r}^{2}}{\hat{\omega}_{r}^{2}} \text { and } \varepsilon_{\underline{\phi}_{r}}(\underline{\theta})=\frac{\left\|\beta_{r}(\underline{\theta}) \underline{\phi}_{r}(\underline{\theta})-\underline{\hat{\phi}}_{r}\right\|}{\left\|\hat{\phi}_{r}\right\|}
$$

be the measures of fit or residuals [16] between the measured modal data and the model predicted modal data for the $r$-th modal frequency and mode shape components, respectively, where $\|\underline{z}\|^{2}=\underline{z}^{\mathrm{T}} \underline{z}$ is the usual Euclidean norm, and $\beta_{r}(\underline{\theta})=\underline{\hat{\phi}}_{r}^{T} \underline{\phi}_{r}(\underline{\theta}) /\left\|\underline{\phi_{r}}(\underline{\theta})\right\|^{2}$ is a normalization constant that guaranties that the measured mode shape $\underline{\phi}_{r}$ at the measured DOFs is closest to the model mode shape $\beta_{r}(\underline{\theta}) \underline{\phi}_{r}(\underline{\theta})$ predicted by the particular value of $\underline{\theta}$. To proceed with the model updating formulation, the measured modal properties are grouped into two groups. The first group contains the modal frequencies while the second group includes the mode shape components for all modes. For each group, a norm is introduced to measure the residuals of the difference between the measured values of the modal properties involved in the group and the corresponding modal values predicted from the model class for a particular value of the parameter set $\underline{\theta}$. For the first group, the measure of fit $J_{1}(\underline{\theta})$ is selected to represent the difference between the measured and the model predicted frequencies for all modes. For the second group, the measure of fit $J_{2}(\underline{\theta})$ is selected to represent the difference between the measured and the model predicted mode shape components for all modes. Specifically, the two measures of fit are given by

$$
J_{1}(\underline{\theta})=\sum_{r=1}^{m} \varepsilon_{\omega_{r}}^{2}(\underline{\theta}) \text { and } J_{2}(\underline{\theta})=\sum_{r=1}^{m} \varepsilon_{\phi_{r}}^{2}(\underline{\theta})=\sum_{r=1}^{m}\left[1-M A C_{r}^{2}(\underline{\theta})\right]
$$

where $\operatorname{MAC}_{r}(\underline{\theta})=\underline{\phi}_{r}{ }^{T} \underline{\phi}_{r}(\underline{\theta}) /\left\|\underline{\phi}_{r}\right\|\left\|\underline{\phi}_{r}\right\|$ is the Modal Assurance Criterion [13] between experimentally identified and estimated mode shapes for the $r$-th mode. Alternative measures of fit can easily be used and found in literature [14-17]. 
Derived from the MAC for any measured frequency point, $\omega_{k}$ a global correlation coefficient may be used [18, 19]:

$$
x_{s}\left(\omega_{k}\right)=\frac{\left|\left\{H_{X}\left(\omega_{k}\right)\right\}^{H}\left\{H_{A}\left(\omega_{k}\right)\right\}\right|^{2}}{\left(\left\{H_{X}\left(\omega_{k}\right)\right\}^{H}\left\{H_{X}\left(\omega_{k}\right)\right\}\right)\left(\left\{H_{A}\left(\omega_{k}\right)\right\}^{H}\left\{H_{A}\left(\omega_{k}\right)\right\}\right)}
$$

where $\left\{H_{X}\left(\omega_{k}\right)\right\}$ and $\left\{H_{A}\left(\omega_{k}\right)\right\}$ are the experimental (measured) and the analytical (predicted) response vectors at matching excitation - response locations. As the MAC value, $x_{s}\left(\omega_{k}\right)$ assumes a value between zero and unity and indicates perfect correlation with $x_{s}\left(\omega_{k}\right)=1$. For $x_{s}\left(\omega_{k}\right)=0$, no correlation exists. Similar to the MAC, $x_{s}\left(\omega_{k}\right)$ is unable to detect scaling errors and is only sensitive to discrepancies in the overall deflection shape of the structure. To emphasis this characteristic, $x_{s}\left(\omega_{k}\right)$ is accordingly called the shape correlation coefficient [19]. The lack of sensitivity to scaling of the shape correlation coefficient does not allow the identification of identical FRFs. This insufficiency becomes even more dramatic if just one measurement and its corresponding prediction are correlated. In this case, the column vectors reduce to scalars and $\left\{H_{A}\left(\omega_{k}\right)\right\}=k\left\{H_{X}\left(\omega_{k}\right)\right\}$ is always satisfied (constant k may be complex), therefore leading to $x_{s}=1$ across the full frequency spectrum for uncorrelated FRFs.

As a result, a supplementary correlation coefficient $x_{a}\left(\omega_{k}\right)$ is proposed by targeting the discrepancies in amplitude. The amplitude correlation coefficient is defined as:

$$
x_{a}\left(\omega_{k}\right)=\frac{2\left|\left\{H_{X}\left(\omega_{k}\right)\right\}^{H}\left\{H_{A}\left(\omega_{k}\right)\right\}\right|}{\left(\left\{H_{X}\left(\omega_{k}\right)\right\}^{H}\left\{H_{X}\left(\omega_{k}\right)\right\}\right)+\left(\left\{H_{A}\left(\omega_{k}\right)\right\}^{H}\left\{H_{A}\left(\omega_{k}\right)\right\}\right)}
$$

where the response vectors are identical to those used for $x_{s}\left(\omega_{k}\right)$. As for the shape correlation coefficient, $x_{a}\left(\omega_{k}\right)$ is defined to lie between zero and unity. This time, however, the correlation measure is more stringent and only becomes unity if $\left\{H_{A}\left(\omega_{k}\right)\right\}=\left\{H_{X}\left(\omega_{k}\right)\right\}$. All elements of the response vectors must be identical in both phase and amplitude even if only one measurement is considered. Similarly, to modal residuals, two measures of fit are proposed using $x_{s}\left(\hat{\omega}_{r}\right)$ and $x_{a}\left(\hat{\omega}_{r}\right)$ which correspond to the identified resonant frequencies of the system:

$$
J_{3}(\underline{\theta})=\sum_{r=1}^{m}\left[1-x_{s}\left(\hat{\omega}_{r}, \underline{\theta}\right)^{2}\right] \text { and } J_{4}(\underline{\theta})=\sum_{r=1}^{m}\left[1-x_{a}\left(\hat{\omega}_{r}, \underline{\theta}\right)^{2}\right]
$$

Minimizing at global minimum the following single objective, traditionally solves the parameter estimation problem:

$$
J(\underline{\theta} ; \underline{w})=w_{1} J_{1}(\underline{\theta})+w_{2} J_{2}(\underline{\theta})+w_{3} J_{3}(\underline{\theta})+w_{4} J_{4}(\underline{\theta})
$$

formed by the four objectives $J_{i}(\underline{\theta})$, using the weighting factors $w_{i} \geq 0, i=1,2,3,4$, with $w_{1}+w_{2}+w_{3}+w_{4}=1$. The objective function $J(\underline{\theta} ; \underline{w})$ represents an overall measure of fit between the measured and the model predicted characteristics. The relative importance of the residual errors in the selection of the optimal model is reflected in the choice of the weights. The results of the identification depend on the weight values used. The optimal solutions for the parameter set $\underline{\theta}$ for given $\underline{w}$ are denoted by $\underline{\hat{\theta}}(\underline{w})[6,20,23]$. 


\section{EXPERIMENTAL APPLICATION}

In this section, the proposed computational framework is applied to update the FE model of the three sections of a real steam turbine, shown in Figure 1. More specifically, the turbine type is LMZ K-300-170 with total power 310MW, which is placed in the unit IV of the thermal power plant in the area of Agios Dimitrios. The turbine consists of three sections, (Low pressure LP, Intermediate Pressure IP, High Pressure HP), which are connected in line. The total mass of the turbine is approximately 76 tones and consisted of 37 stages (10 stages in Low pressure section, 17 stages in Intermediate pressure section and 10 stages in High pressure section) and of 27 different types of blades (total number of blades 4126). The low pressure section consisted from the same blade types used in the intermediate section. The 27 different blade types are presented in Figure 2.

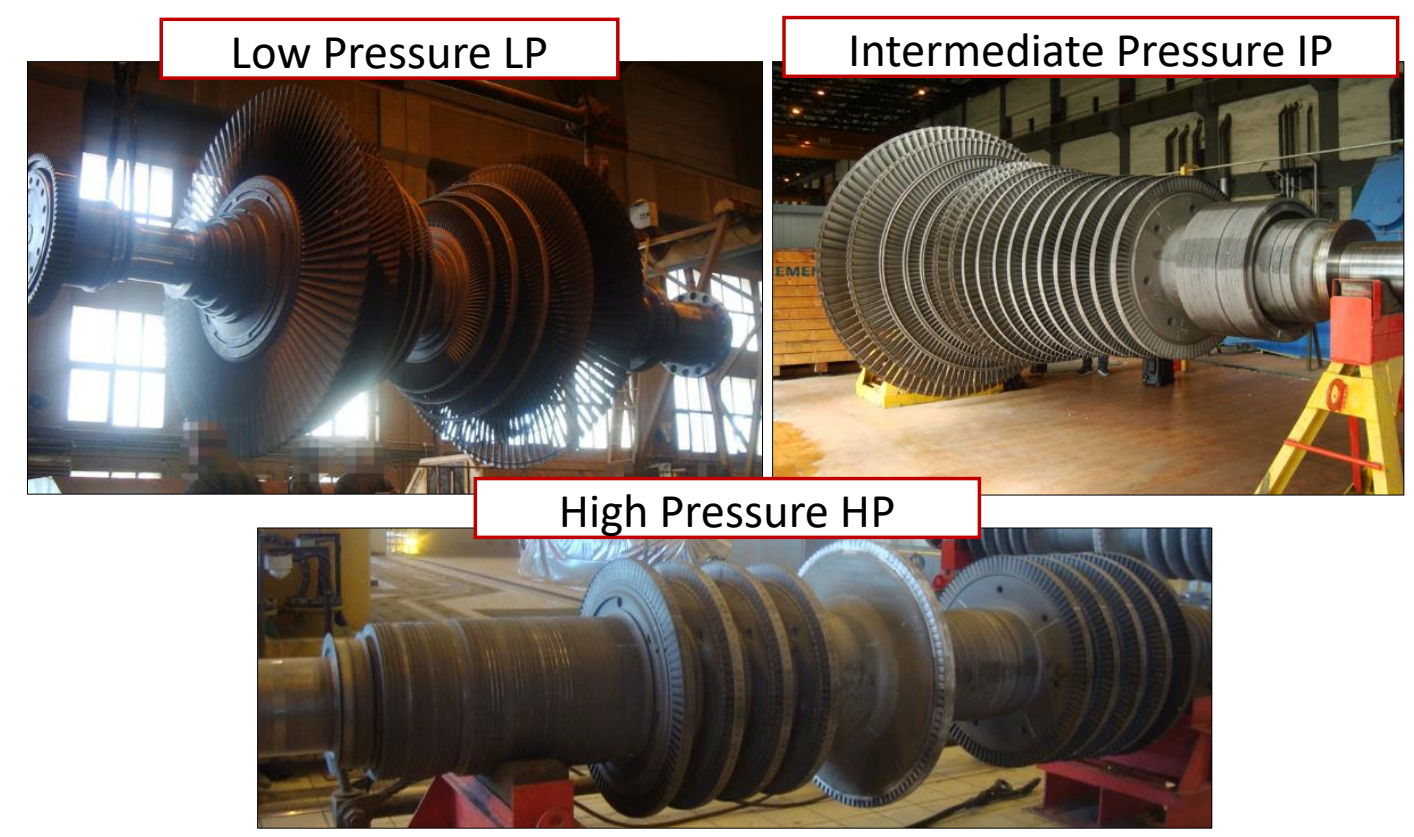

Figure 1: The three sections of a real steam turbine, (a) low pressure turbine, (b) intermediate pressure turbine and, (c) high pressure turbine.

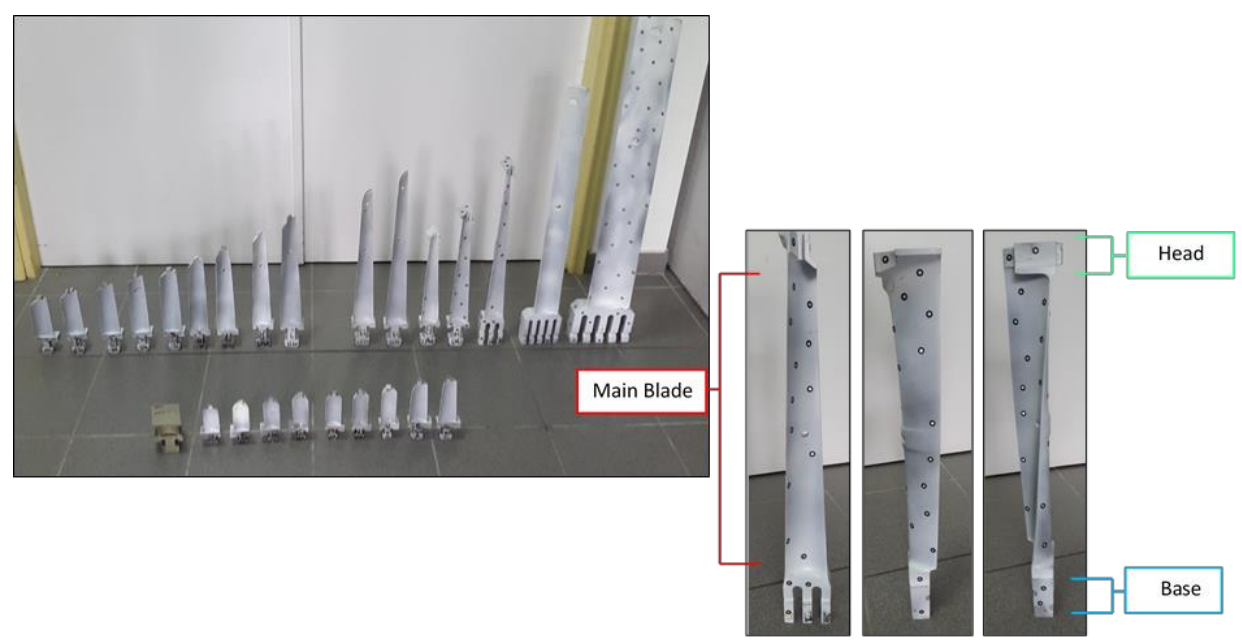

Figure 2: The 27 different blade types of the three sections of a real steam turbine 


\subsection{Digitization and CAD Model of the Steam Turbine}

Due to large size of the results of the complete steam rotor, is selected to present the basic idea of the applied method only for the intermediate pressure rotor. First, exploiting a 3D Laser Scanner, the digital shape of each blade type which used in the three turbine sections was developed by using the DSR (Digital Shape Reconstruction) method. In this process, four basic steps are being followed in order to collect, process and design the final CAD model. First, the geometrical data of each blade were captured, exploiting the 3D scanner's functionalities, as well as its software tools in order to produce a primary stereo-lithography (STL) file. As a second step, compatible utilities were used to pre-process the initial raw model in order to create the final STL file of the digitized geometry, before designing the CAD surfaces [6, 24-26]. Next, in order to produce the initial CAD model, a segmentation of the triangulated STL model and NURBS (Non-uniform Rational B-Splines) surface fitting, were applied. The whole procedure is depicted in Figure 3, for one blade of the section IP15 of the intermediate pressure section.

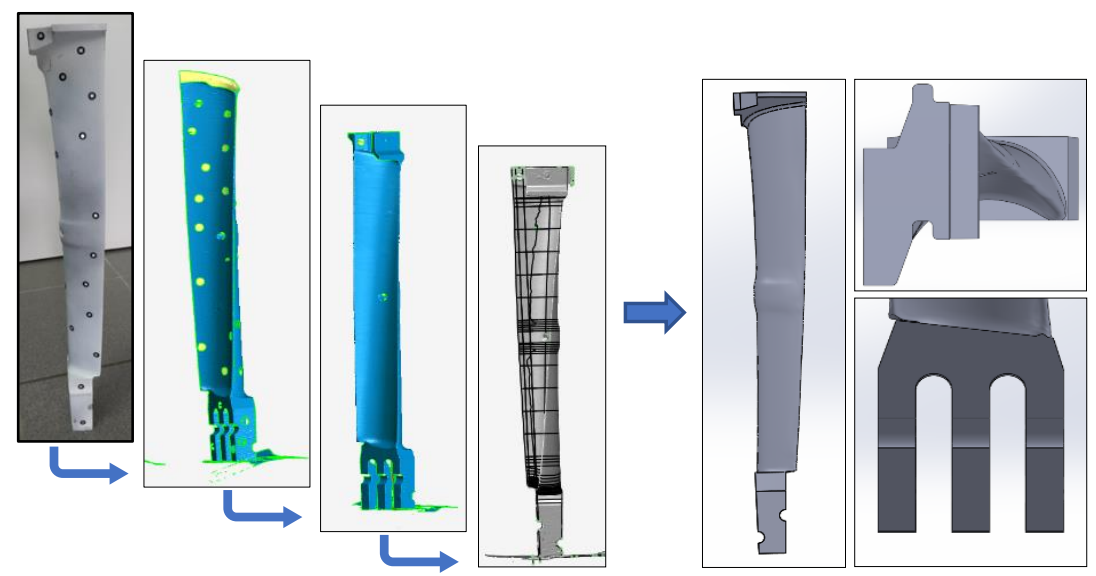

Figure 3: Digitization and final 3D CAD model of one blade of the section IP15 of the intermediate pressure section.

The geometry of the rotor shaft is also unknown. The 3D CAD model of the rotor shaft was developed using the digital 2D drawings (Figure 4).
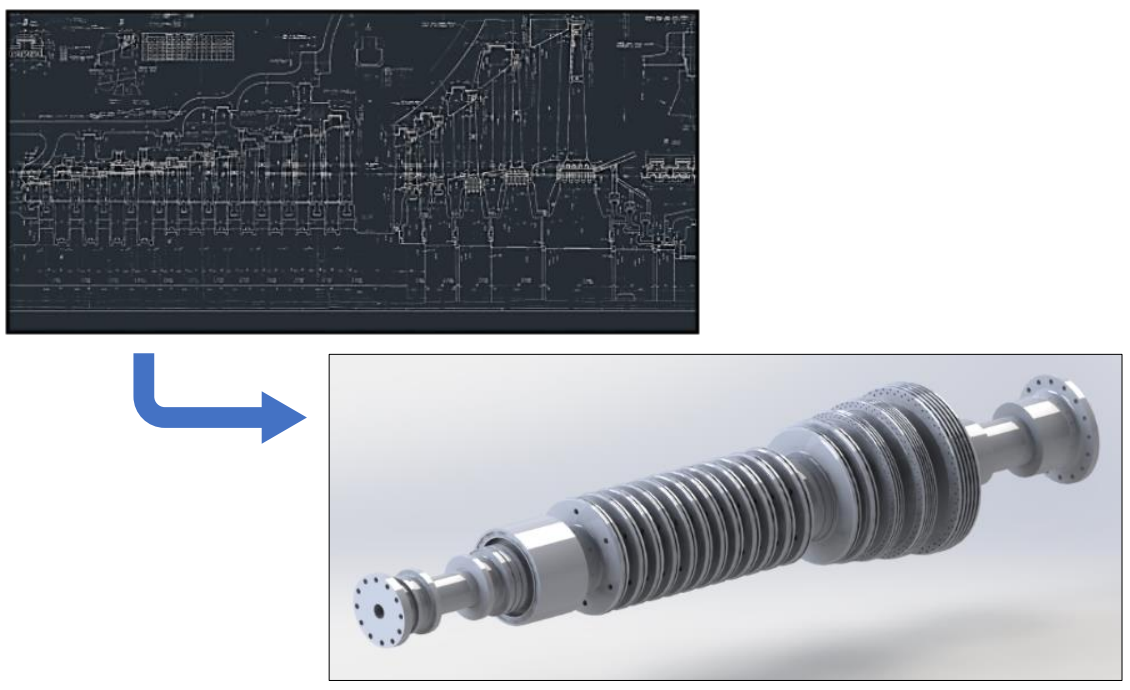

Figure 4: Digitization and final 3D CAD model of the rotor shaft of the intermediate pressure section. 
The complete 3D CAD assembly of the intermediate pressure section, including the rotor shaft and the blades of all the 17 sections is presented in Figure 5.

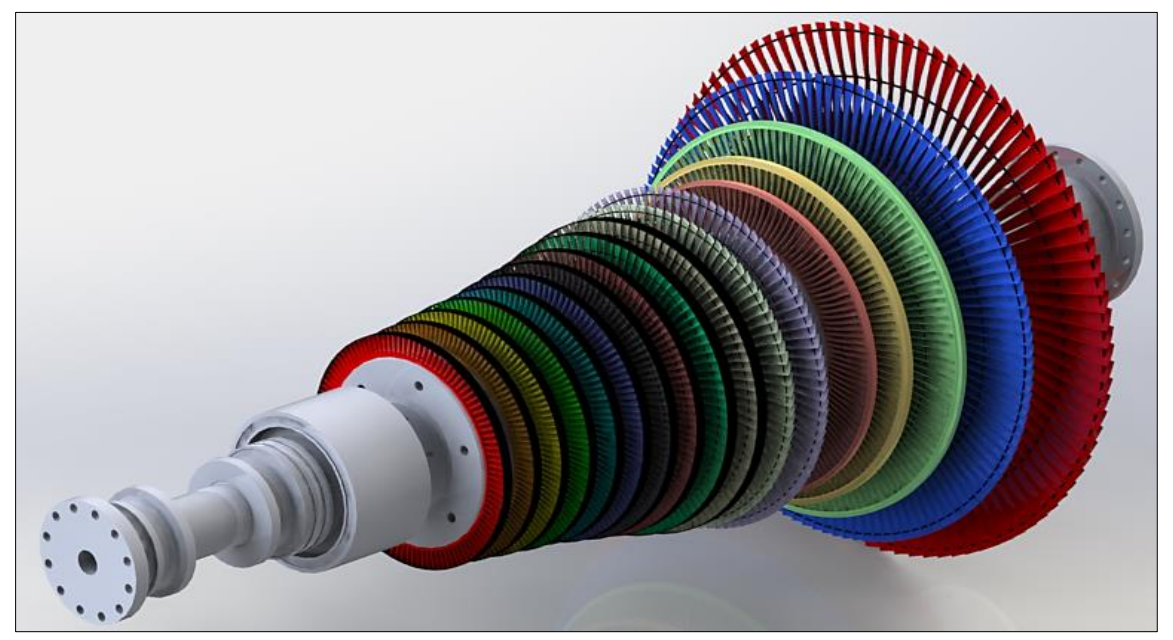

Figure 5: Complete 3D CAD assembly of the intermediate pressure section, including the rotor shaft and the blades of all the 17 sections.

Following the same procedure was carried out for the other two sections (low and high pressure) and, the final 3D CAD model of the complete steam turbine was developed (Figure 6).

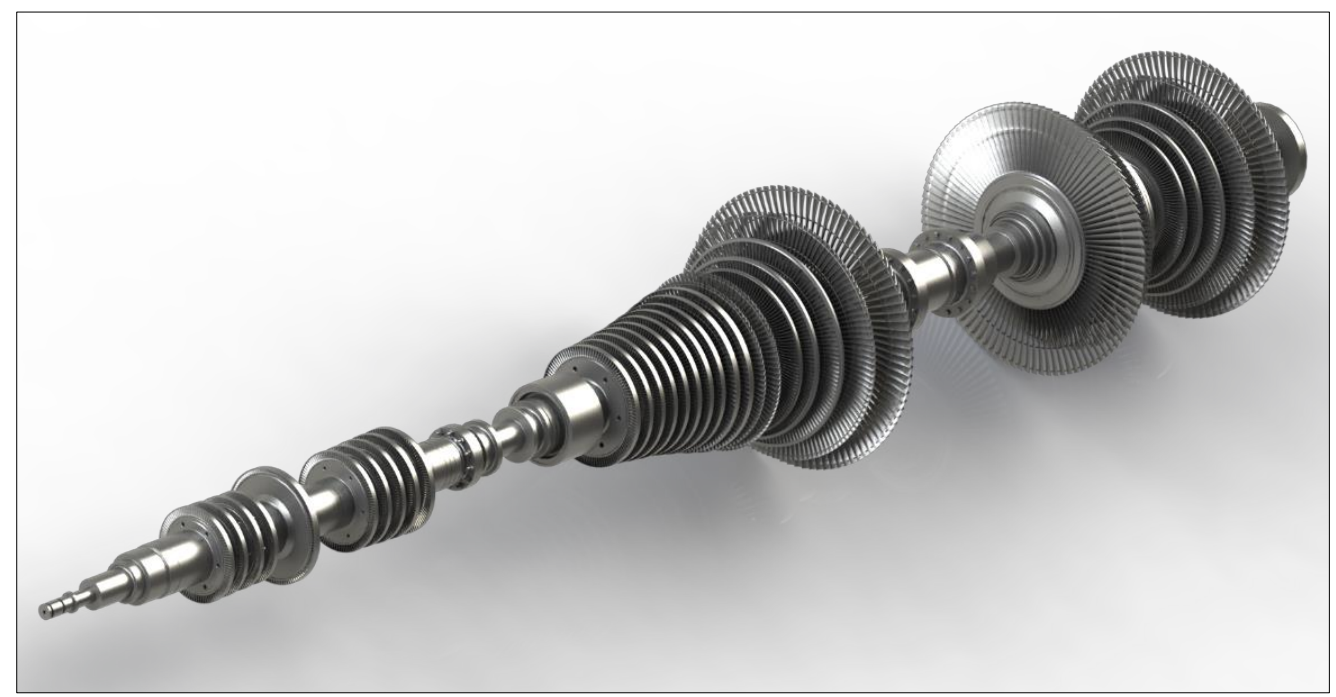

Figure 6: Final 3D CAD of the complete steam turbine.

\subsection{Finite Element Model}

The geometry of the turbine sections discretized by solid elements (tetrahedral). Due to complex geometry of the structure, the total number of degrees of freedom of the resulting complete rotor model is about fifty-five million degrees of freedom $(55,000,000)$. The detailed FE Model of the frame presented in Figure 7. For the development and solution of the finite element model some appropriate software was used [27, 28]. Typical eigenmodes of the three sections and of the complete model presented in Figure 8. 


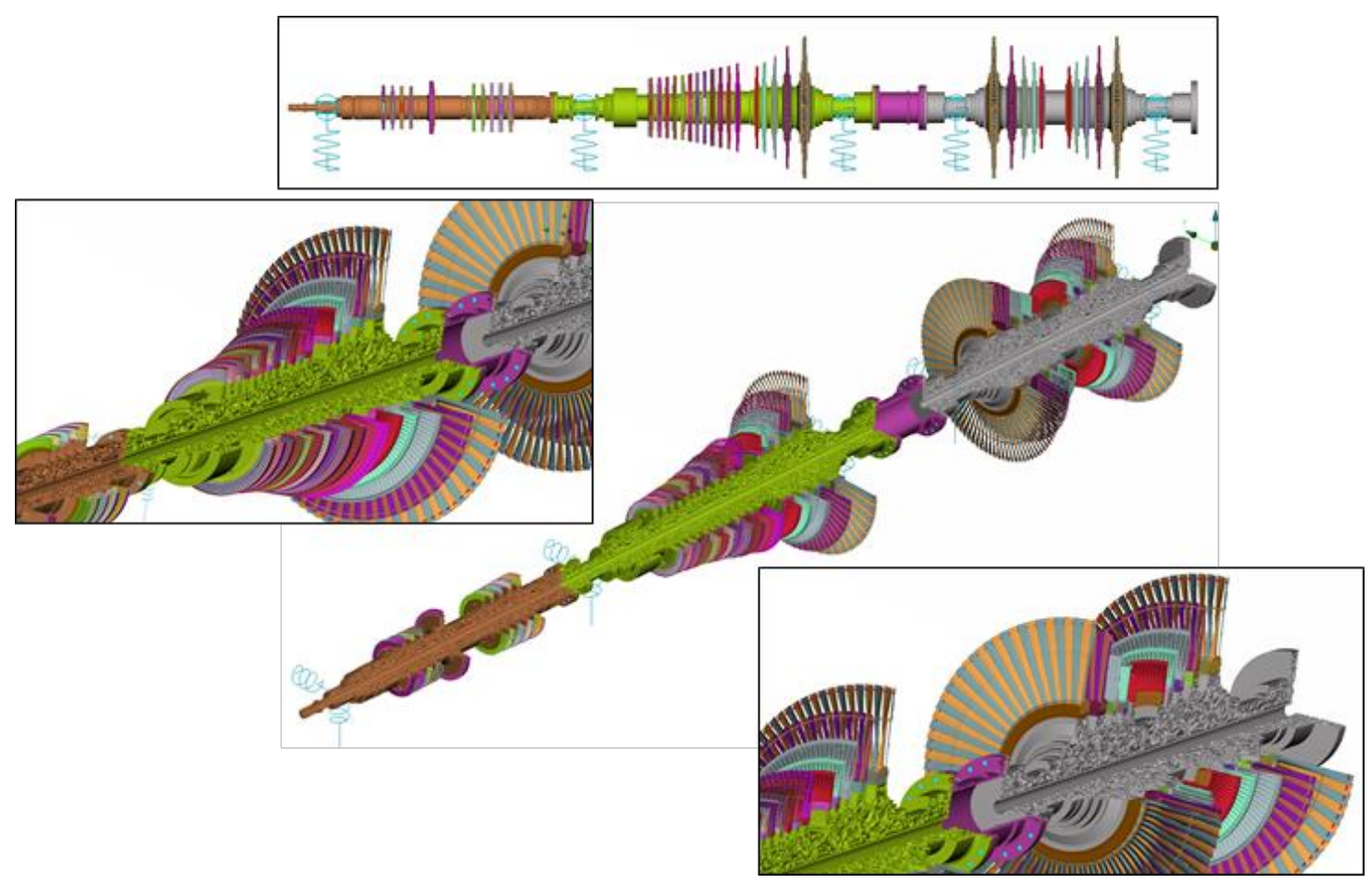

Figure 7: Final finite element model of the complete steam turbine.
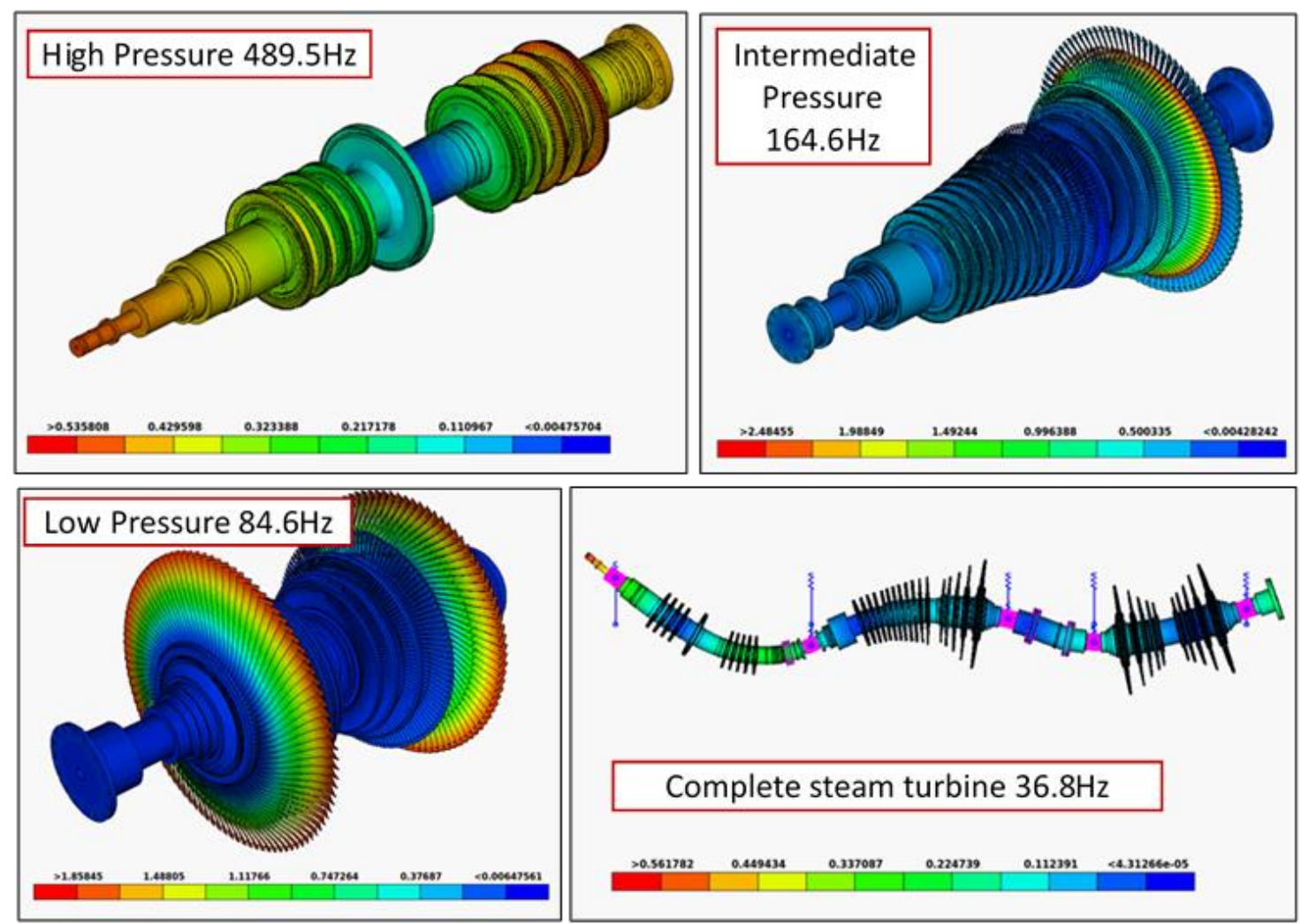

Figure 8: Typical eigenmodes of the three sections and of the complete model. 


\subsection{Experimental Modal Analysis}

Next, in order to quantify the dynamic characteristics of the turbine, an experimental modal analysis of the three turbine sections was performed. Again, due to large size of the results, is selected to present the results only for the intermediate pressure rotor. The rotor, was hung up with the help of a crane and straps, to approximate free-free boundary conditions for the test.

First, all the necessary elements of the FRF matrix required for determining the response of the rotor were determined by imposing impulsive loading $[6-10,12]$. The measured frequency range was $0-2048 \mathrm{~Hz}$, which includes the analytical frequency range of interest, $0-700 \mathrm{~Hz}$. A schematic illustration of the measurement geometry for the modal analysis of the intermediate pressure rotor, with the real experimental set up of this test is presented Figure 9. For instance, Figure 10 shows the magnitude of typical elements of the FRF matrix.

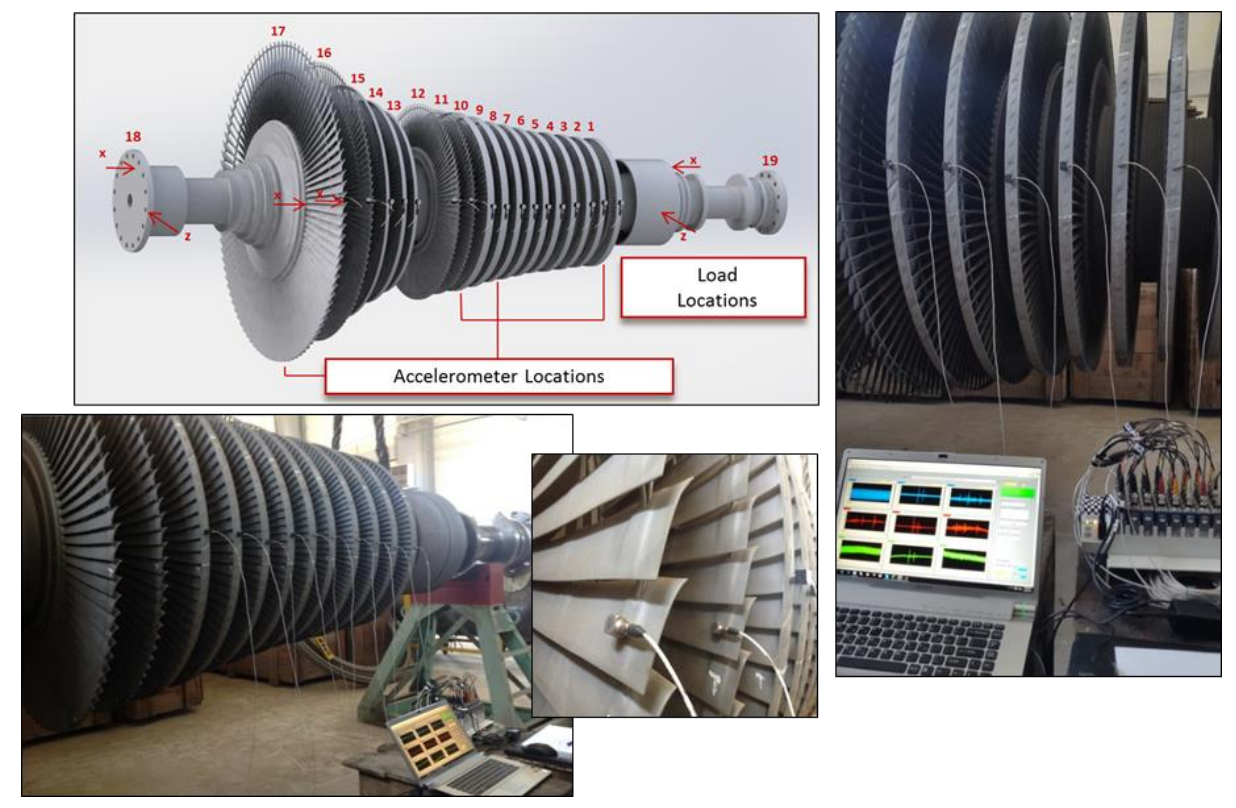

Figure 9: Schematic illustration of the measurement geometry and real experimental set-up for the modal analysis of the intermediate pressure rotor.

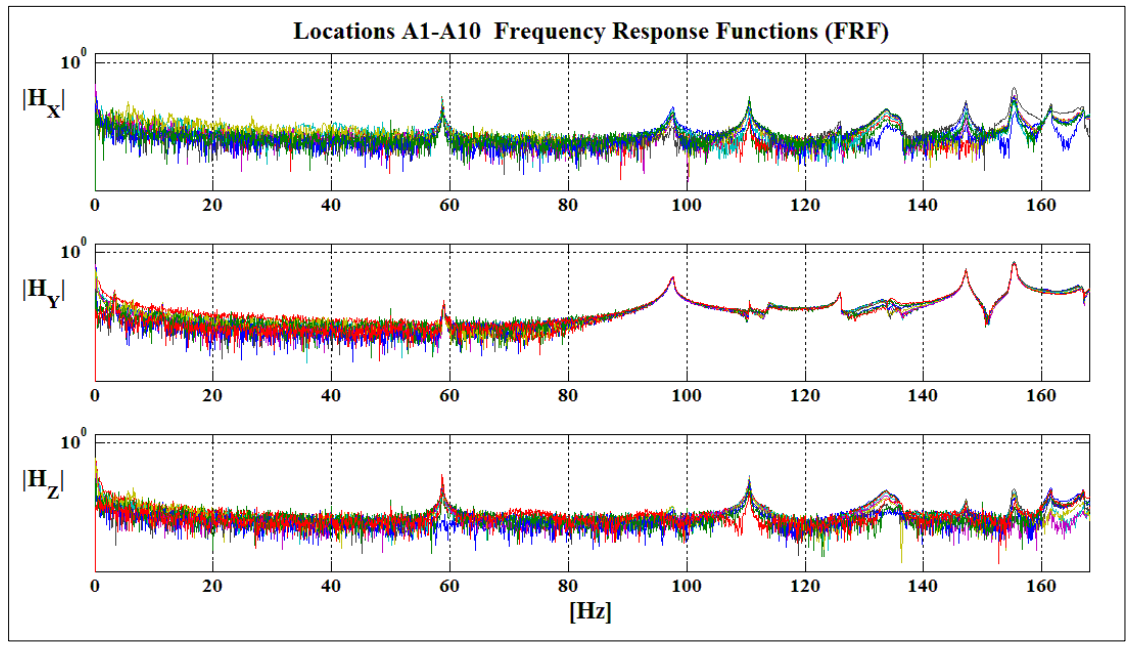

Figure 10: Typical elements of the experimental FRF matrix for the intermediate pressure rotor. 
Based on the measured FR functions, the natural frequencies and the damping ratios of the rotor were estimated by applying the "Rational Fraction Polynomial Method" (RFPM). This method has certain attractive merits, especially for systems with high modal density, like the system under consideration [11, 22, 29]. The identified mode shapes have also been recorded so that they can be used for updating the finite element models. As an outcome of the above procedure, the first column of Table 1 presents the values of the lowest 11 natural frequencies $\left(\omega_{r E}\right)$ of the frame, while the corresponding damping ratios are included in the fourth column. In the same table, the second column presents the values of the natural frequencies obtained from the analysis of the nominal finite element model $\left(\omega_{r N_{F E}}\right)$ and the third column compares these frequencies with the corresponding frequencies obtained by the experimental data.

\begin{tabular}{|c|c|c|c|c|}
\hline Mode & $\begin{array}{l}\text { Identified } \\
\text { Modal } \\
\text { Frequency }\end{array}$ & $\begin{array}{l}\text { Nominal FE } \\
\text { Predicted } \\
\text { Modal } \\
\text { Frequency }\end{array}$ & $\begin{array}{c}\text { Difference } \\
\text { between } \\
\text { Identified and FE } \\
\text { Predicted Modal } \\
\text { Frequencies }\end{array}$ & $\begin{array}{c}\text { Identified } \\
\text { Modal } \\
\text { Damping } \\
\text { Ratio }\end{array}$ \\
\hline & $\omega_{r E}[H z]$ & $\omega_{r N_{F E}}[H z]$ & $\frac{\omega_{r N_{r z}}-\omega_{r E}}{\omega_{r N_{r z}}} 100 \%$ & $\zeta_{r E}(\%)$ \\
\hline 1 & 58.70 & 63.04 & 6.88 & 0.21 \\
\hline 2 & 101.30 & 89.52 & 13.16 & 0.19 \\
\hline 3 & 110.60 & 127.22 & 13.06 & 0.12 \\
\hline 4 & 126.10 & 135.62 & 7.02 & 0.08 \\
\hline 5 & 134.80 & 143.45 & 6.03 & 0.13 \\
\hline 6 & 135.01 & 145.41 & 7.15 & 0.13 \\
\hline 7 & 142.32 & 156.12 & 8.84 & 0.12 \\
\hline 8 & 147.40 & 158.23 & 6.84 & 0.16 \\
\hline 9 & 156.77 & 171.22 & 8.44 & 0.16 \\
\hline 10 & 161.30 & 181.36 & 11.06 & 0.35 \\
\hline 11 & 167.70 & 183.42 & 8.57 & 0.23 \\
\hline
\end{tabular}

Table 1: Modal frequencies and modal damping ratios for the intermediate pressure rotor.

\section{FINITE ELEMENT MODEL UPDATING}

\subsection{FE model parameterization}

The parameterization of the finite element model of the intermediate section is introduced in order to demonstrate the applicability of the proposed finite element model updating method. This model consists of about twenty-three million degrees of freedom. The parameterized model consisting of forty-four parts which is shown in Figure 11. At each of these parts are used as design variables the Young's modulus and the density. Thus, the final number of the design parameters are eighty-eight (88) variables.

The initial values of material properties for all parts was set at $7,850 \mathrm{Kg} / \mathrm{m}^{3}$ for the density and at 210GPa for the Young's modulus. The upper and lower limit, which were selected to be used for the optimization process was selected in $\pm 10 \%$ of the initial values.

The finite element model is updated using the lowest eleven identified modal frequencies and mode shapes shown in Table 1. The identified mode shapes include components at all 17 sensor locations. Additionally, we define as design response and total weight of the model, in order to be taken into consideration during the optimization process.

The results from the FE model updating method are shown in Table 2. In this table presented a comparison between identified $\left(\omega_{r E}\right)$ and optimal FE predicted modal frequencies $\left(\omega_{r O_{F E}}\right)$. 


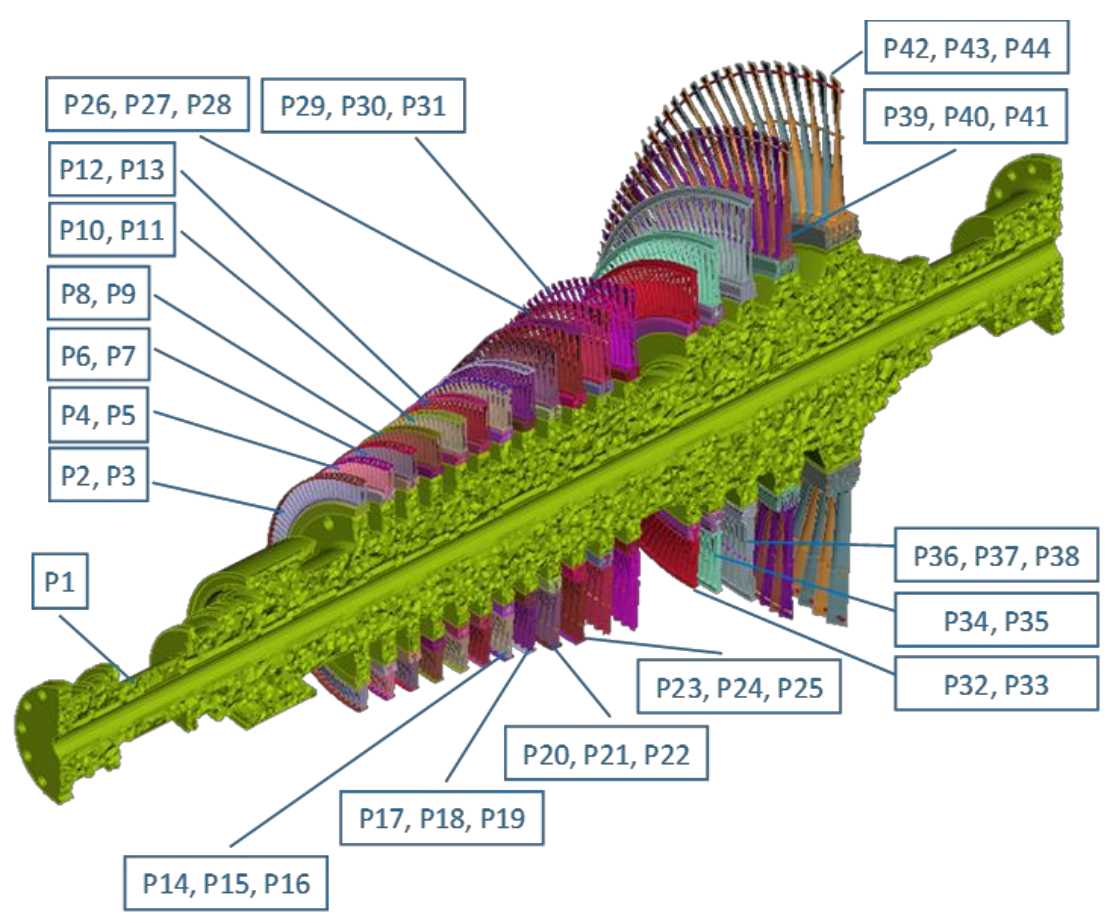

Figure 11: Parts of the parameterized FE model of the intermediate pressure rotor.

\begin{tabular}{cccc}
\hline Mode & $\begin{array}{c}\text { Identified } \\
\text { Modal } \\
\text { Frequency }\end{array}$ & $\begin{array}{c}\text { Optimal FE } \\
\text { Predicted } \\
\text { Modal } \\
\text { Frequency }\end{array}$ & $\begin{array}{c}\text { Difference } \\
\text { between Identified } \\
\text { and FE Predicted } \\
\text { Modal Frequencies }\end{array}$ \\
\hline & $\omega_{r E}[H z]$ & $\omega_{r O_{F E}}[H z]$ & $\frac{\omega_{r O_{F E}}-\omega_{r E}}{\omega_{r O_{F E}}} 100 \%$ \\
\hline $\mathbf{1}$ & 58.70 & 59.45 & 1.26 \\
$\mathbf{2}$ & 101.30 & 99.62 & 1.69 \\
$\mathbf{3}$ & 110.60 & 112.22 & 1.44 \\
$\mathbf{4}$ & 126.10 & 128.62 & 1.96 \\
$\mathbf{5}$ & 134.80 & 134.85 & 0.04 \\
$\mathbf{6}$ & 135.01 & 135.92 & 0.67 \\
$\mathbf{7}$ & 142.32 & 144.24 & 1.33 \\
$\mathbf{8}$ & 147.40 & 148.64 & 0.83 \\
$\mathbf{9}$ & 156.77 & 154.22 & 1.65 \\
$\mathbf{1 0}$ & 161.30 & 160.04 & 0.79 \\
$\mathbf{1 1}$ & 167.70 & 164.54 & 1.92 \\
\hline
\end{tabular}

Table 2: Comparison between identified and optimal FE predicted modal frequencies for the intermediate pressure rotor.

\section{SUMMARY}

The applicability and effectiveness of a computational framework namely the model reduction method, model updating method and experimental modal analysis, is explored by calibrating a high-fidelity FE model of a steam turbine rotor with fifty-five million degrees of freedom. First, using a reverse engineering method, the digital shape of the three sections of a steam turbine rotor was developed and the final parametric CAD model was created. The finite 
element model of the turbine were created using tetrahedral solid elements. Modal analysis techniques were applied in order to identify the modal parameters. Direct comparison of the numerical and experimental data verified the reliability and accuracy of the methodology applied.

\section{REFERENCES}

[1] Abella, R.J., J.M. Daschbach, and R.J. McNichols, Reverse engineering industrial applications. Computers \& Industrial Engineering, 1994. 26(2): p. 381-385.

[2] Dagli, C.H. and M.A. Idowu, Instantaneous Modelling and Reverse Engineering of DataConsistent Prime Models in Seconds! Procedia Computer Science Complex Adaptive Systems San Jose, CA November 2-4, 2015, 2015. 61: p. 373-380.

[3] Moroni, G., et al., Toward a Methodological Knowledge based Approach for Partial Automation of Reverse Engineering. 24th CIRP Design Conference, 2014. 21: p. 270275.

[4] Várudy, T., et al., Reverse Engineering of Geometric ModelsReverse engineering of geometric models - an introduction. Computer-Aided Design, 1997. 29(4): p. 255-268.

[5] Wang, J., et al., A framework for 3D model reconstruction in reverse engineering. Computers \& Industrial Engineering, 2012. 63(4): p. 1189-1200.

[6] <A Matlab Package for Analysis and Solution of Discrete Ill-Posed Problems.pdf $>$.

[7] Ewins, D.J., Modal Testing: Theory and Practice. 1984, Somerset, England: Research Studies Press.

[8] Giagopoulos, D. and S. Natsiavas, Hybrid (numerical-experimental) modeling of complex structures with linear and nonlinear components. Nonlinear Dynamics, 2007. 47(1): p. 193-217.

[9] Giagopoulos, D. and S. Natsiavas, Dynamic Response and Identification of Critical Points in the Superstructure of a Vehicle Using a Combination of Numerical and Experimental Methods. Experimental Mechanics, 2015. 55(3): p. 529-542.

[10] Mohanty, P. and D.J. Rixen, Identifying mode shapes and modal frequencies by operational modal analysis in the presence of harmonic excitation. Experimental Mechanics, 2005. 45(3): p. 213-220.

[11] Richardson, M.H. and D.L. Formenti, Global curve fitting of frequency response measurements using the rational fraction polynomial method, in Third IMAC Conference. 1985: Orlando, Florida.

[12] Spottswood, S.M. and R.J. Allemang, On the Investigation of Some Parameter Identification and Experimental Modal Filtering Issues for Nonlinear Reduced Order Models. Experimental Mechanics, 2007. 47(4): p. 511-521.

[13] Allemang, R.J. and D.L. Brown, A Correlation Coefficient for Modal Vector Analysis, in 1st International Modal Analysis Conference. 1982: Orlando, USA.

[14] Moaveni, B., et al., Damage Identification of a Composite Beam Using Finite Element Model Updating. Computer-Aided Civil and Infrastructure Engineering, 2008. 23(5): p. 339-359. 
[15] Moaveni, B., et al., Damage identification study of a seven-story full-scale building slice tested on the UCSD-NEES shake table. Structural Safety, 2010. 32(5): p. 347-356.

[16] Weber, B., P. Paultre, and J. Proulx, Structural damage detection using nonlinear parameter identification with Tikhonov regularization. Structural Control and Health Monitoring, 2007. 14(3): p. 406-427.

[17] Yuen, K.-V., J.L. Beck, and L.S. Katafygiotis, Efficient model updating and health monitoring methodology using incomplete modal data without mode matching. Structural Control and Health Monitoring, 2006. 13(1): p. 91-107.

[18] Grafe, H., Model updating of large structural dynamics models using measured response function, in Department of Mechanical Engineering. 1999, Imperial College: London.

[19] Grafe, H., Review of frequency response function updating methods. 1995, BRITEURANUS BRE2-CT94-0946.

[20] Christodoulou, K., et al., Structural model updating and prediction variability using Pareto optimal models. Computer Methods in Applied Mechanics and Engineering, 2008. 198(1): p. 138-149.

[21] Giagopoulos, D., et al., Bayesian Uncertainty Quantification and Propagation in Nonlinear Structural Dynamics, in Topics in Model Validation and Uncertainty Quantification, Volume 5: Proceedings of the 31st IMAC, A Conference on Structural Dynamics, 2013, T. Simmermacher, et al., Editors. 2013, Springer New York: New York, NY. p. 33-41.

[22] Ntotsios, E. and C. Papadimitriou, Multi-objective optimization algorithms for finite element model updating, in International Con- ference on Noise and Vibration Engineering (ISMA2008). 2008: Katholieke Universiteit Leuven, Leuven, Belgium. p. 66-80.

[23] Papadimitriou, C., et al., Variability of updated finite element models and their predictions consistent with vibration measurements. Structural Control and Health Monitoring, 2012. 19(5): p. 630-654.

[24] Béchet, E., J.C. Cuilliere, and F. Trochu, Generation of a finite element MESH from stereolithography (STL) files. Computer-Aided Design, 2002. 34(1): p. 1-17.

[25] Bianconi, F., Bridging the gap between CAD and CAE using STL files. International Journal of CAD/CAM, 2002. 2(1): p. 55-67.

[26] Rypl, D. and Z. Bittnar, Generation of computational surface meshes of STL models. Journal of Computational and Applied Mathematics, 2006. 192(1): p. 148-151.

[27] BETA CAE Systems, S.A., ANSA, EPILYSIS \& META-Post. 2016, BETA CAE Systems, S.A.: Thessaloniki, Greece.

[28] DTECH, DYNAMIS 3.1.1. 2013, DTECH: Thessaloniki, Greece.

[29] Friswell, M.I. and J.E.T. Penny, Updating model parameters from frequency domain data via reduced order models. Mechanical Systems and Signal Processing, 1990. 4(5): p. 377391. 